\title{
DVFS-Aware Consolidation for Energy-Efficient Clouds
}

\author{
Patricia Arroba ${ }^{a b}$ (Advisors: José M. Moya ${ }^{a b}$, José L. Ayala ${ }^{c}$, Rajkumar Buyya ${ }^{d}$ ) \\ ${ }^{a}$ Laboratorio de Sistemas Integrados (LSI), Universidad Politécnica de Madrid, Madrid, Spain. \\ ${ }^{b} \mathrm{CCS}$ - Center for Computational Simulation, Campus de Montegancedo UPM, Boadilla del Monte, Spain. \\ ${ }^{c}$ DACYA, Complutense University of Madrid, Spain. \\ ${ }^{d}$ CLOUDS Laboratory, The University of Melbourne, Australia.
}

Motivation. Computational demand on data centers is increasing due to the growing popularity of Cloud applications. However, data centers are becoming unsustainable in terms of power consumption and growing energy costs so they must be placed on a more scalable curve. Recently, there has been a growing interest in developing techniques to provide power management in Clouds. Dynamic Voltage and Frequency Scaling (DVFS) helps to reduce the consumption of underutilized resources dynamically, while consolidation strategies decrease significantly the static consumption by reducing the number of active servers, thus increasing their utilization. However, DVFS is traditionally applied locally, regardless the consolidation techniques. Understanding the relationship between power, DVFS and consolidation is crucial to enable new energy-efficient strategies that combine these effective techniques. To this purpose, the dependency of power on some traditionally ignored factors like frequency or static consumption, which are increasingly influencing the consumption patterns of these infrastructures, must now be considered. Furthermore, as Cloud services are provided under strict Service Level Agreement (SLA) conditions, power consumption in data centers may be minimized, taking into account a trade-off between DVFS and performance, without violating the SLA requirements whenever it is feasible. Also, Cloud workloads vary significantly over time, difficulting the optimal allocation of resources that requires a tradeoff between consolidation and performance. Therefore, the implementation of consolidation policies that are aware of both DVFS and energy consumption while considering QoS has the potential to optimize the sustainability of Cloud data centers.

Proposed Solution. In this work we aim to find an energy optimization strategy for Cloud data centers that combines DVFS and consolidation techniques. Our policy is not only aware of the utilization of the incoming workload to be assigned, but also is conscious of the impact of its allocation in terms of frequency. One of the main challenges when designing data center optimizations is to implement fast algorithms that can be evaluated during runtime. For this reason, our research is focused on the design of an optimization algorithm that is simple in terms of computational requirements, in which both decision making and its execution in a real infrastructure are fast. The proposed algorithm is based on a

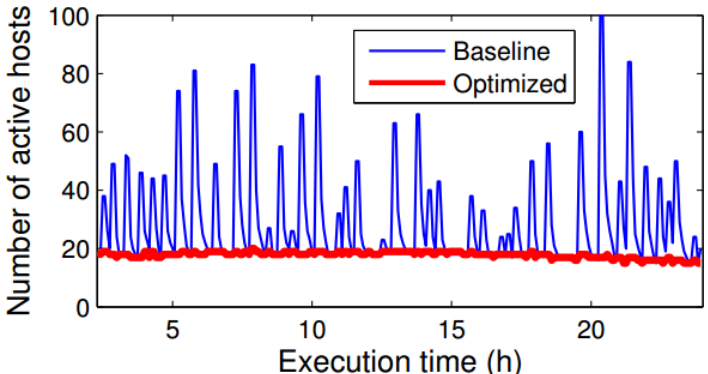

Fig. 1: Number of active hosts during runtime

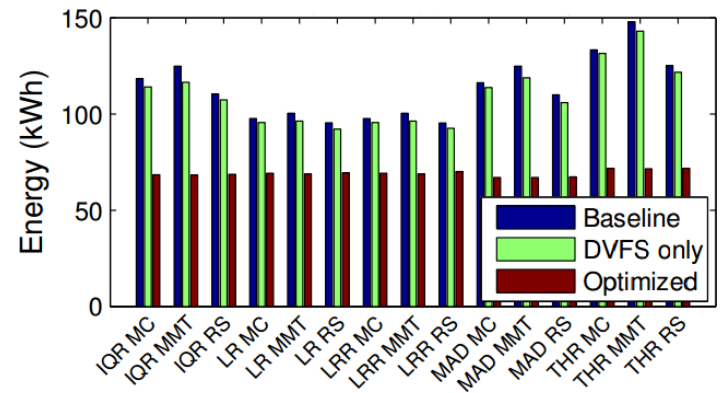

Fig. 2: Average energy consumption comparison per test

bin packing problem [1] where servers are represented as bins with variable sizes due to the frequency scaling. To design our optimization technique, we first characterize performance and power contributions in terms of those architectural parameters most influenced by DVFS and consolidation [2]. The obtained power model offers an accuracy of about $4.46 \%$ and allows a better understanding of how energy varies depending on frequency and utilization simultaneously. In our proposed optimization, VMs are consolidated in those hosts that have a high utilization but, on the contrary, have a low increase in frequency due to the utilization increment. This approach minimizes the number of bins used by this combinatorial NPhard problem while taking full advantage of the range of CPU utilization available for each frequency.

Results. We have performed an extensive evaluation on CloudSim [3] that represents accurately the modeling of virtualized data centers. Our application framework consists of real Cloud traces from the global research network PlanetLab [4]. Hosts are modeled as Fujitsu RX300-S6 servers 
based on Intel Xeon E5620@2.4GHz, virtualized by the QEMU-KVM hypervisor. VM instances correspond to existing types of Amazon EC2. To evaluate the performance of our frequency-aware optimization we compare our work with two different approaches where the frequency is not considered during consolidation: the Baseline and the DVFS-only scenarios, where the DVFS is switched off and on respectively. The three provided scenarios, including our frequency-aware optimization, Freq-Aware, are tested for 15 different tests, each of them representing a specific combination of overloading detection and VM selection algorithms in the consolidation process.

Our algorithm speeds up consolidation and the elastic scale out of the IT infrastructure, presenting a global utilization increase of up to $23.46 \%$ by reducing the number of active hosts by $44.91 \%$ (see Figure 11. Our strategy reduces the number of VM migrations by $22.17 \%$ and by $19.61 \%$ when compared with Baseline and DVFS-only. This behavior impacts on the energy usage of the data center, where the consumption of both Baseline and DVFS-only grows at a higher rate during dynamic workload variations than for Freq-Aware scenario. We achieve competitive energy savings of $37.86 \%$ and $35.72 \%$ in average respectively (see Figure 2), maintaining QoS, even improving slightly SLA violations around $0.01 \%$.

Novelty and Contributions. The key contribution of our work is a novel frequency-aware consolidation algorithm that reduces the energy consumption of the data center while maintaining its QoS. The algorithm is light, scalable and offers an elastic scale-out under varying demand of resources, making it suitable for quickly adaptation to workload fluctuations in the data center. We have performed an extensive evaluation using real Cloud traces and an accurate power model based on data gathered from real servers. Our results demonstrate that including DVFS awareness in workload management provides substantial energy savings of up to $51.62 \%$ for scenarios under dynamic workload conditions.

\section{REFERENCES}

[1] A. Beloglazov and R. Buyya, "Optimal online deterministic algorithms and adaptive heuristics for energy and performance efficient dynamic consolidation of virtual machines in cloud data centers," Concurr. Comput. : Pract. Exper, vol. 24, no. 13, pp. 1397-1420, Sep. 2012.

[2] P. Arroba, J. L. Risco-Martín, M. Zapater, J. M. Moya, J. L. Ayala, and K. Olcoz, "Server power modeling for runtime energy optimization of cloud computing facilities," Energy Procedia, vol. 62, no. 0, pp. 401 - 410, 2014.

[3] R. N. Calheiros, R. Ranjan, A. Beloglazov, C. A. F. De Rose, and R. Buyya, "Cloudsim: A toolkit for modeling and simulation of cloud computing environments and evaluation of resource provisioning algorithms," Softw. Pract. Exper., vol. 41, no. 1, pp. 23-50, Jan. 2011.

[4] K. Park and V. S. Pai, "Comon: A mostly-scalable monitoring system for planetlab," SIGOPS Oper. Syst. Rev., vol. 40, no. 1, pp. 65-74, Jan. 2006. 